\title{
Social Optimization and Pricing Policy in Cognitive Radio Networks with an Energy Saving Strategy
}

\author{
Shunfu Jin, ${ }^{1,2}$ Si Chen, ${ }^{1,2}$ and Jinliang Zhang ${ }^{3}$ \\ ${ }^{1}$ College of Information Science and Engineering, Yanshan University, Qinhuangdao 066004, China \\ ${ }^{2}$ Key Laboratory for Computer Virtual Technology and System Integration of Hebei Province, Yanshan University, \\ Qinhuangdao 066004, China \\ ${ }^{3}$ National Center for Public Cultural Services, Ministry of Culture of China, Beijing 110000, China
}

Correspondence should be addressed to Shunfu Jin; jsf@ysu.edu.cn

Received 10 January 2016; Accepted 7 June 2016

Academic Editor: George Ghinea

Copyright (C) 2016 Shunfu Jin et al. This is an open access article distributed under the Creative Commons Attribution License, which permits unrestricted use, distribution, and reproduction in any medium, provided the original work is properly cited.

The rapid growth of wireless application results in an increase in demand for spectrum resource and communication energy. In this paper, we firstly introduce a novel energy saving strategy in cognitive radio networks (CRNs) and then propose an appropriate pricing policy for secondary user (SU) packets. We analyze the behavior of data packets in a discrete-time singleserver priority queue under multiple-vacation discipline. With the help of a Quasi-Birth-Death (QBD) process model, we obtain the joint distribution for the number of SU packets and the state of base station (BS) via the Matrix-Geometric Solution method. We assess the average latency of SU packets and the energy saving ratio of system. According to a natural reward-cost structure, we study the individually optimal behavior and the socially optimal behavior of the energy saving strategy and use an optimization algorithm based on standard particle swarm optimization (SPSO) method to search the socially optimal arrival rate of SU packets. By comparing the individually optimal behavior and the socially optimal behavior, we impose an appropriate admission fee to SU packets. Finally, we present numerical results to show the impacts of system parameters on the system performance and the pricing policy.

\section{Introduction}

With the explosive growth of wireless application, one of the hot topics of research is how to improve the spectrum efficiency as well as reduce the communication consumption. Cognitive radio is a recent and promising development in wireless communications technology [1]. Green energy powered cognitive radio networks (CRNs) are capable of liberating wireless communications from spectral and energy constraints [2].

One of the key challenges in wireless networks is how to control the base station (BS) in order to decrease the energy consumption. In addition, we should also focus on how to ensure the Quality of Service (QoS) of end users and spectrum efficiency. From the related literature, we find that the main method to release the energy constraint is sleep mode, by which communication power can be reduced when the BS is at the sleep state $[3,4]$. We also find that the cognitive radio technology has been studied as an effective solution to alleviate the limitation of the spectrum resource [5]. In addition, in order to achieve the maximum value of social profit of the system, people pay more and more attention to the behaviors of the data packets, including the individually optimal behavior and the socially optimal behavior $[6,7]$.

In this context, we firstly introduce an energy saving strategy with sleep mode in CRNs. The proposed strategy highlights how the BS is switched between the sleep state and the awake state to achieve the trade-off between the energy saving effect and the response performance. In addition, we assess the system performance by constructing a QuasiBirth-Death (QBD) process model and validate the analytical results with simulations. According to a natural reward-cost structure, we analyze the individually optimal behavior and the socially optimal behavior of the energy saving strategy and use an optimization algorithm based on standard particle swarm optimization (SPSO) method to search the socially 
optimal arrival rate of secondary user (SU) packets. By comparing the individually optimal behavior and the socially optimal behavior, we impose an appropriate admission fee to SU packets.

The remainder of this paper is organized as follows. In Section 3, we describe an energy saving strategy with sleep mode and system model, and then we carry out the stability condition of the system. In Section 4, we analyze the model and derive the formulas for the performance measures. In Section 5, we demonstrate the influence of system parameters on the system performance with numerical results. In Section 6, through theoretical analysis and numerical comparison of the individually optimal behavior and the socially optimal behavior of the energy saving strategy, we impose an appropriate pricing policy. Finally, we summarize the conclusions in Section 7.

\section{Related Works}

In wireless networks, sleep mode is one of key techniques to reduce energy consumption. One important way to address the energy saving problem is to introduce sleep mode to the BS. Dini et al. studied the sleep mode as an approach to decrease the energy consumption in BS of long term evolution (LTE) heterogeneous networks (HetNets). Furthermore, they introduced two sleep algorithms, namely, single-sleep and multiple-sleep, to determine the time instant to enable micro- or pico-BSs to employ sleep mode [3]. Rengarajan et al. characterized the maximum energy saving that can be achieved in a cellular wireless access network with sleep mode under a given performance constraint. By taking into account the QoS perceived by end users, their approach allows the derivation of more realistic estimates that can be used to evaluate the efficacy of schemes utilizing sleep modes to save energy [4]. Li et al. proposed a new sleep mechanism called sleep-transmit mode (STM) that provides a solution by adding a transmit state to the currently employed mechanism in passive optical network (PON) standards. When compared to interrupted sleep mode, STM achieves up to $29 \%$ power reduction while providing comparable delay performance for upstream packets [8]. Some authors used a Markov chain technique to evaluate the energy savings with the sleep mode mechanism in a single user terminal $[9,10]$. In contrast to $[9,10]$, we are more concerned with the sleep mode on the BS (rather than that on the user terminal). In [11], Premalatha et al. took a survey on energy saving methods for green communication networks and found that sleep mode is adapted for reducing the energy consumption of BS.

Recently, the opportunistic spectrum access mechanism in CRNs has been paid more attention to make the spectrum scarcity less severe that wireless communications face now. Cognitive radio is a form of wireless communication in which a transceiver can intelligently detect the communication channels which are in use and which are not and instantly move into unused channels while avoiding occupied ones [12]. Cognitive radio is widely considered to resolve the scarcity of spectrum bands and to meet the burgeoning requirements of wireless services by employing opportunistic spectrum sharing [13]. In [14], Anwar et al. proposed a new optimization-based access strategy of multipacket reception (MPR) channel for multiple SUs accessing the primary user (PU) spectrum. The concept of CRNs is regarded as a prosperous technology duo to high spectrum efficiency [1214]. In addition to efficient spectrum usage, how to reduce energy consumption is the current problem to be solved. An energy harvesting CRN design will not only ease the spectrum shortage problem, but also result in a green design [15]. Most of the existing dynamic spectrum access (DSA) schemes only consider the SUs' transmission in licensed spectrum without considering the unlicensed bands. In order to reduce dropping and blocking probabilities of SUs, the researchers extended classical schemes, including random access scheme and reservation based channel access scheme [16].

The application of game theory in performance optimization is becoming more and more widespread and important. Cuong et al. studied a noncooperative game problem for $M / M / 1$ queueing control in the cognitive radio system. In queueing game with server breakdowns, each customer wants to optimize its benefit in a selfish distributed manner [17]. Zheng et al. proposed a distributed cooperative framework to improve the energy efficiency of green cellular networks. Based on the traffic load, neighboring BSs cooperate to optimize the BS switching (sleeping) strategies so as to maximize the energy saving while guaranteeing users' minimal service requirements [18]. Li and Han studied the individually and socially optimal strategies based on queueing control in cognitive radio systems. The study result reveals that the individually optimal strategy does not yield the socially optimal one. To bridge the gap between the individually and socially optimal strategies, a pricing mechanism is proposed to toll the service of each SU [7]. In CRNs, Tran et al. studied price-based spectrum access control, which characterized network operators' service provisions to delay-sensitive SUs via pricing strategies [19].

\section{Energy Saving Strategy with Sleep Mode and Model Description}

3.1. Energy Saving Strategy with Sleep Mode. In CRNs, the PU has high priority to occupy the spectrum. If the spectrum sensing results are perfect, the transmission of a PU packet will not be influenced by SU packets, while interrupting the transmission of an SU packet by PU packets is possible. The SU packet will queue at the head of the buffer for future transmission.

The BS in conventional CRNs is always awake even though there are not any packets, either PU packet or SU packet, to be transmitted or received. In this paper, we describe an energy saving strategy. In order to reduce the energy consumption, the BS will be switched into the sleep state from the awake state when the spectrum is idle and the buffer of SU packets is empty.

In the proposed energy saving strategy, the BS will be switched between two states, namely, sleep state and awake state, respectively.

(1) During the sleep state, the BS will wake up for a short time at every boundary of the time slot to 
listen whether there is a packet arrival or not. If there is no packet arrival before the sleep timer expires, the BS will start another sleep period once the sleep timer is over. If a PU packet arrives at the system during the sleep state, the sleep timer will be stopped immediately, and the BS will be switched to the awake state. If there is no PU packet arrival but at least one SU packet arrival during the sleep state, the BS will be switched to the awake state once the sleep timer expires.

(2) If the BS is in the awake state, all the packets will be transmitted continuously. PU packets will be transmitted with high priority, while SU packets will be transmitted opportunistically. When all the packets are transmitted completely, a sleep timer will be started and the BS will be switched to the sleep state.

We note that the energy consumption is lower when the BS is in the sleep state than that in the awake state. By this way, communication energy will be saved in CRNs with the sleep mode. As a cost of energy conservation, the SU packets will be delayed for longer time before transmission. It is necessary for us to evaluate and optimize the system performance mathematically.

3.2. Model Description. We consider a CRN with a single licensed channel. The energy saving strategy proposed above is adopted by this system.

We consider a system model in discrete-time field. Time is assumed to be divided into fixed-length intervals, referred to as slots. The slots are marked as $n(n=1,2, \ldots)$. We consider an early arrival system (EAS). In other words, we suppose that the arrival of data packets occurs at the beginning instant of a slot, marked as $\left(n, n^{+}\right)$, while the departure of data packets occurs at the end of instant of a slot, marked as $\left(n^{-}, n\right)$. We also assume that the data packets are transmitted within the slots, and the state transition of the BS occurs at the instant $n^{+}$.

We assume that the arriving intervals of PU and SU packets follow geometric distributions with parameters $\lambda_{\mathrm{pu}}(0<$ $\left.\lambda_{\mathrm{pu}}<1, \bar{\lambda}_{\mathrm{pu}}=1-\lambda_{\mathrm{pu}}\right)$ and $\lambda_{\mathrm{su}}\left(0<\lambda_{\mathrm{su}}<1, \bar{\lambda}_{\mathrm{su}}=1-\lambda_{\mathrm{su}}\right)$, respectively. In other words, the arrival rates of PU and SU packets are $\lambda_{\mathrm{pu}}$ and $\lambda_{\mathrm{su}}$, respectively. We assume that the transmission times of a PU packet and an SU packet follow geometric distributions with parameters $\mu_{\mathrm{pu}}\left(0<\mu_{\mathrm{pu}}<\right.$ $\left.1, \bar{\mu}_{\mathrm{pu}}=1-\mu_{\mathrm{pu}}\right)$ and $\mu_{\mathrm{su}}\left(0<\mu_{\mathrm{su}}<1, \bar{\mu}_{\mathrm{su}}=1-\mu_{\mathrm{su}}\right)$, respectively. Without loss of generality, we did not assume $\mu_{\mathrm{su}}=\mu_{\mathrm{pu}}$ when describing the system model. PU packets are transmitted with pure loss and preemptive priority, while SU packets are transmitted with opportunity and interrupt retrial. In addition, we assume that the sleep timer length follows a geometric distribution with sleeping parameter $\theta$.

Let $X_{n}=i(i=0,1,2, \ldots)$ be the total number of SU packets at the instant $n^{+}$and let $Y_{n}=j(j=0,1,2)$ be the state of the BS at the instant $n^{+}(j=0$ means the BS is in the sleep state, $j=1$ means the BS is awake and a PU packet is being transmitted, and $j=2$ means the BS is awake and an SU packet is being transmitted). According to the above descriptions, a two-dimensional process composed of the total number $X_{n}$ of SU packets and the state $Y_{n}$ of the $\mathrm{BS}$ is established. The state space of $\left\{\left(X_{n}, Y_{n}\right), n \geq 1\right\}$ is given as follows:

$$
\mathbf{\Omega}=\{(i, j): i \geq 0, j=0,1,2\} .
$$

Let $\pi_{i, j}$ be the probability that the number of SU packets is $i$ and the state of the BS is $j$ at the steady state. $\pi_{i, j}$ is given as follows:

$$
\begin{aligned}
\pi_{i, j}=\lim _{n \rightarrow \infty} P\left\{X_{n}=i, Y_{n}=j\right\} & \\
& i=0,1,2, \ldots, j=0,1,2 .
\end{aligned}
$$

Let $\pi_{\mathbf{k}}$ be steady-state probability vector that the number of SU packets is $k . \pi_{\mathbf{k}}$ is given by

$$
\pi_{\mathbf{k}}=\left(\pi_{k, 0}, \pi_{k, 1}, \pi_{k, 2}\right), \quad k \geq 0 .
$$

Let $\Pi$ be the probability distribution of the system in steady state. $\Pi$ is then given by

$$
\Pi=\left(\pi_{0}, \pi_{1}, \pi_{2}, \ldots\right) .
$$

3.3. Stability Condition. In the system considered in this paper, there are two types of packets, namely, PU packets and SU packets. When the total traffic load $\rho$ for both the PU packets and SU packets is less than 1 , the system will reach a stable state.

We firstly address the traffic load $\rho_{\mathrm{pu}}$ of PU packets, which is defined as the probability that the $\mathrm{BS}$ is occupied by $\mathrm{PU}$ packets.

In CRNs, PU packets are transmitted with preemptive priority. Under the assumption that the spectrum sensing is perfect, the transmission of PU packets is independent of that of SU packets. Recall that once a PU packet arrives at the system, no matter the BS is in sleep state or not, the PU packet will be transmitted immediately. That is to say, the transmission of PU packets is also independent of the sleep mode. For this reason, the activities of PU packets can be modeled as a simple two-state Markov chain.

From the perspective of PU packets, the BS has two states, namely, idle state and busy state. The idle state means that the BS is not occupied by PU packets, while the busy state means that the BS is occupied by PU packets. Let $\pi_{\text {idle }}$ be the probability that the BS is in idle state and let $\pi_{\text {busy }}$ be the probability that the BS is in busy state. The BS changes to busy state from idle state with probability $\lambda_{\mathrm{pu}}$; that is, there is an arrival of PU packet. The probability that the BS changes to idle state from busy state is $\mu_{\mathrm{pu}} \bar{\lambda}_{\mathrm{pu}}$; that is, the PU packet is successfully transmitted, and no PU packet arrives at the system. We can get the balance equation and the normalization condition as

$$
\begin{aligned}
\pi_{\text {idle }} \lambda_{\text {pu }} & =\pi_{\text {busy }} \mu_{\text {pu }} \bar{\lambda}_{\mathrm{pu}}, \\
\pi_{\text {idle }}+\pi_{\text {busy }} & =1 .
\end{aligned}
$$


It is straight forward to derive $\pi_{\mathrm{idle}}$ and $\pi_{\text {busy }}$ as follows:

$$
\begin{gathered}
\pi_{\text {idle }}=\frac{\mu_{\mathrm{pu}} \bar{\lambda}_{\mathrm{pu}}}{\lambda_{\mathrm{pu}}+\mu_{\mathrm{pu}} \bar{\lambda}_{\mathrm{pu}}}, \\
\pi_{\text {busy }}=\frac{\lambda_{\mathrm{pu}}}{\lambda_{\mathrm{pu}}+\mu_{\mathrm{pu}} \bar{\lambda}_{\mathrm{pu}}} .
\end{gathered}
$$

From the definition of the traffic load $\rho_{\mathrm{pu}}$ of PU packets, we know that the traffic load $\rho_{\mathrm{pu}}$ of PU packets is the probability that the $\mathrm{BS}$ is in busy state. So

$$
\rho_{\text {pu }}=\pi_{\text {busy }}
$$

We define the traffic load $\rho_{\mathrm{su}}$ of SU packet as the probability that the BS is occupied by SU packets. Since there is a buffer with infinite capacity for SU packets, no SU packets can be blocked. From the perspective of SU packets, once an SU packet enters the system, this SU packet will not leave the system until it is successfully transmitted. So the traffic load $\rho_{\text {su }}$ of SU packets is given as follows:

$$
\rho_{\mathrm{su}}=\frac{\lambda_{\mathrm{su}}}{\mu_{\mathrm{su}}} .
$$

Combining (7) and (8), we can derive the total traffic load $\rho$ as follows:

$$
\rho=\rho_{\mathrm{pu}}+\rho_{\mathrm{su}}=\frac{\lambda_{\mathrm{pu}}}{\lambda_{\mathrm{pu}}+\mu_{\mathrm{pu}} \bar{\lambda}_{\mathrm{pu}}}+\frac{\lambda_{\mathrm{su}}}{\mu_{\mathrm{su}}} .
$$

The stability condition of the system is shown as follows:

$$
\rho<1
$$

that is,

$$
\frac{\lambda_{\mathrm{pu}}}{\lambda_{\mathrm{pu}}+\mu_{\mathrm{pu}} \bar{\lambda}_{\mathrm{pu}}}+\frac{\lambda_{\mathrm{su}}}{\mu_{\mathrm{su}}}<1 .
$$

We assume this stability condition to be fulfilled in the remainder of the paper.

\section{Model Analysis and Performance Measures}

4.1. Model Analysis. We define $\mathbf{P}$ as the one step state transition probability matrix of the two-dimensional process $\left\{\left(X_{n}, Y_{n}\right), n \geq 1\right\}$. According to the number of SU packets, we give the one step state transition probability matrix $\mathbf{P}$ in a block structure as follows:

$$
\mathbf{P}=\left(\begin{array}{cccccc}
\mathbf{B}_{00} & \mathbf{B}_{01} & & & & \\
\mathbf{B}_{10} & \mathbf{A}_{1} & \mathbf{A}_{0} & & & \\
& \mathbf{A}_{2} & \mathbf{A}_{1} & \mathbf{A}_{0} & & \\
& & \mathbf{A}_{2} & \mathbf{A}_{1} & \mathbf{A}_{0} & \\
& & & \ddots & \ddots & \ddots
\end{array}\right),
$$

where

$$
\begin{aligned}
& \mathbf{B}_{00}=\bar{\lambda}_{\mathrm{su}}\left(\begin{array}{ccc}
\bar{\lambda}_{\mathrm{pu}} & \lambda_{\mathrm{pu}} & 0 \\
\mu_{\mathrm{pu}} \bar{\lambda}_{\mathrm{pu}} & \bar{\mu}_{\mathrm{pu}}+\mu_{\mathrm{pu}} \lambda_{\mathrm{pu}} & 0 \\
0 & 0 & 0
\end{array}\right) \text {, } \\
& \mathbf{B}_{01}=\lambda_{\mathrm{su}}\left(\begin{array}{ccc}
\bar{\lambda}_{\mathrm{pu}} \bar{\theta} & \lambda_{\mathrm{pu}} & \bar{\lambda}_{\mathrm{pu}} \theta \\
0 & \bar{\mu}_{\mathrm{pu}}+\mu_{\mathrm{pu}} \lambda_{\mathrm{pu}} & \mu_{\mathrm{pu}} \bar{\lambda}_{\mathrm{pu}} \\
0 & 0 & 0
\end{array}\right) \text {, } \\
& \mathbf{B}_{10}=\mu_{\mathrm{su}} \bar{\lambda}_{\mathrm{su}}\left(\begin{array}{ccc}
0 & 0 & 0 \\
0 & 0 & 0 \\
\bar{\lambda}_{\mathrm{pu}} & \lambda_{\mathrm{pu}} & 0
\end{array}\right) \text {, } \\
& \mathbf{A}_{0}=\mathbf{B}_{r c}=\lambda_{\mathrm{su}}\left(\begin{array}{ccc}
\bar{\lambda}_{\mathrm{pu}} \bar{\theta} & \lambda_{\mathrm{pu}} & \bar{\lambda}_{\mathrm{pu}} \theta \\
0 & \bar{\mu}_{\mathrm{pu}}+\mu_{\mathrm{pu}} \lambda_{\mathrm{pu}} & \mu_{\mathrm{pu}} \bar{\lambda}_{\mathrm{pu}} \\
0 & \bar{\mu}_{\mathrm{su}} \lambda_{\mathrm{pu}} & \bar{\mu}_{\mathrm{su}} \bar{\lambda}_{\mathrm{pu}}
\end{array}\right) \text {, } \\
& 1 \leq r<c,
\end{aligned}
$$

$\mathbf{A}_{1}=\mathbf{B}_{r c}$

$$
\begin{gathered}
=\left(\begin{array}{ccc}
\bar{\lambda}_{\mathrm{su}} & 0 & 0 \\
0 & \bar{\lambda}_{\mathrm{su}} & 0 \\
0 & 0 & \bar{\mu}_{\mathrm{su}} \bar{\lambda}_{\mathrm{su}}+\mu_{\mathrm{su}} \lambda_{\mathrm{su}}
\end{array}\right) \\
\times\left(\begin{array}{ccc}
\bar{\lambda}_{\mathrm{pu}} \bar{\theta} & \lambda_{\mathrm{pu}} & \bar{\lambda}_{\mathrm{pu}} \theta \\
0 & \bar{\mu}_{\mathrm{pu}}+\mu_{\mathrm{pu}} \lambda_{\mathrm{pu}} & \mu_{\mathrm{pu}} \bar{\lambda}_{\mathrm{pu}} \\
0 & \lambda_{\mathrm{pu}} & \bar{\lambda}_{\mathrm{pu}}
\end{array}\right)
\end{gathered}
$$$$
r=c \geq 1
$$

$$
\mathbf{A}_{2}=\mathbf{B}_{r c}=\mu_{\mathrm{su}} \bar{\lambda}_{\mathrm{su}}\left(\begin{array}{ccc}
0 & 0 & 0 \\
0 & 0 & 0 \\
0 & \lambda_{\mathrm{pu}} & \bar{\lambda}_{\mathrm{pu}}
\end{array}\right), \quad r>c \geq 1
$$

The component matrix $\mathbf{B}_{r c}(r, c \geq 0)$ represents the case that the number of SU packets in the system changes to $c$ from $r$. Particularly, we redefine three special cases that if $r=c \geq 1$, $\mathbf{B}_{r c}$ is denoted by $\mathbf{A}_{1}$, if $1 \leq r<\mathcal{c}, \mathbf{B}_{r c}$ is denoted by $\mathbf{A}_{0}$, and if $r>c \geq 1, \mathbf{B}_{r c}$ is denoted by $\mathbf{A}_{2}$.

The structure of $\mathbf{P}$ shows that the system transition occurs only in adjacent levels. Moreover, it is clear that the rows of the transition probability matrix $\mathbf{P}$ are repeating after the third row. From the book written by Neuts [20], we know that the matrix $\mathbf{P}$ has a block-tridiagonal structure which indicates that $\left\{\left(X_{n}, Y_{n}\right), n \geq 1\right\}$ is a QBD process.

For the QBD process of the transition probability matrix $\mathbf{P}$, the necessary and sufficient condition of $\left\{\left(X_{n}, Y_{n}\right), n \geq 1\right\}$ to be positive recurrence is that the matrix quadratic equation

$$
\mathbf{R}^{2} \mathbf{A}_{2}+\mathbf{R} \mathbf{A}_{1}+\mathbf{A}_{0}=\mathbf{R}
$$


has a minimal nonnegative solution $\mathbf{R}$ and the spectral radius $\mathrm{SP}(\mathbf{R})<1$ and the six-dimensional stochastic matrix

$$
B[\mathbf{R}]=\left(\begin{array}{cc}
\mathbf{B}_{00} & \mathbf{B}_{01} \\
\mathbf{B}_{10} & \mathbf{A}_{1}+\mathbf{R} \mathbf{A}_{2}
\end{array}\right)
$$

has left invariant vector. When $\left\{\left(X_{n}, Y_{n}\right), n \geq 1\right\}$ is positive recurrent, its stationary distribution satisfies

$$
\begin{aligned}
\boldsymbol{\pi}_{k} & =\boldsymbol{\pi}_{1} \mathbf{R}^{k-1}, \quad k \geq 1, \\
\left(\boldsymbol{\pi}_{0}, \boldsymbol{\pi}_{1}\right) & =\left(\boldsymbol{\pi}_{0}, \boldsymbol{\pi}_{1}\right) B[\mathbf{R}], \\
\boldsymbol{\pi}_{0} \mathbf{e}+\boldsymbol{\pi}_{1}(\mathbf{I}-\mathbf{R})^{-1} \mathbf{e} & =1,
\end{aligned}
$$

where $\mathbf{e}$ is a three-dimensional column vector with all elements being equal to one.

For analyzing this model, we need to find the minimal nonnegative solution $\mathbf{R}$ of matrix (14). In fact, it is difficult to find an analytical solution for this system and therefore a numerical solution is required. In this case, we usually need to derive the recursion expression of the rate matrix $\mathbf{R}$.

Firstly, from (14), we derive the iterative expressions as follows:

$$
\mathbf{R}_{n+1}=\left(\mathbf{R}_{n}^{2} \mathbf{A}_{2}+\mathbf{A}_{0}\right)\left(\mathbf{I}-\mathbf{A}_{1}\right)^{-1}, \quad n=0,1, \ldots,
$$

where $\mathbf{R}_{n}$ is the $n$th order approximation of $\mathbf{R}$ and $\mathbf{R}_{0}$ is the initial value of $\mathbf{R}$. Then, we can obtain $\mathbf{R}$ by a recursive algorithm in Algorithm 1.

Algorithm 1 (algorithm to obtain the minimal nonnegative solution $\mathbf{R})$.

Step 1. Set the initial number of iterations by $n=0$, the initial value $\mathbf{R}$ by $\mathbf{R}_{0}=\mathbf{0}$, the error precision $\varepsilon$, where $\varepsilon$ is greater than 0 , but infinitely close to 0 .

Step 2. Calculate the expression of $\mathbf{R}_{n+1}$ by matrix (17).

Step 3. Update $\mathbf{R}^{*}$ by $\mathbf{R}^{*}=\mathbf{R}_{n+1}-\mathbf{R}_{n}$.

If $\max \left\{\left|\mathbf{R}^{*}\right|\right\}>\varepsilon$, then $n=n+1$, goto Step 2 .

else $\mathbf{R}=\mathbf{R}_{n+1}$.

Step 4. Output R.

With the help of the Matrix-Geometric Solution method and the recursive algorithm in Algorithm 1, we can get the steady-state probability $\pi_{i, j}$ defined in (2).

4.2. Performance Measures. In this subsection, we derive two system performance measures in terms of the average latency and the energy saving ratio.

We first consider the average latency of SU packets, denoted by $\bar{W}_{\text {su }}$, which is defined as the time duration from the instant at which an SU packet joins the system to the instant that the SU packet is successfully transmitted. With
TABLE 1: System parameters in numerical results.

\begin{tabular}{lc}
\hline Parameter & Value \\
\hline Carrier frequency & $2.4 \mathrm{GHz}$ \\
Data transmission rate & $39 \mathrm{Mbps}$ \\
Unit time slot & $1 \mathrm{~ms}$ \\
Energy consumption per slot in sleep state & $1 \mathrm{~mJ}$ \\
Energy consumption per slot in awake state & $6 \mathrm{~mJ}$ \\
Energy consumption for each listening procedure & $2 \mathrm{~mJ}$ \\
Service rate of SU packets $\mu_{\mathrm{su}}$ & 0.75 \\
Service rate of PU packets $\mu_{\mathrm{pu}}$ & 0.65 \\
\hline
\end{tabular}

Little's formula, the average latency $\bar{W}_{\text {su }}$ of SU packets is given as follows:

$$
\bar{W}_{\mathrm{su}}=\frac{1}{\lambda} \sum_{\mathrm{su}} \sum_{i=0}^{\infty} \sum_{j=0}^{2} i \pi_{i, j} .
$$

Next, we derive the energy saving ratio $\delta$, which is defined as the reduction of the energy consumption per slot due to the introduction of the sleep mode. Energy is consumed normally in the awake state and is saved in the sleep state. Furthermore, additional energy will be consumed for each listening procedure in the proposed energy saving strategy. Therefore, the energy saving ratio $\delta$ is given as follows:

$$
\begin{aligned}
\delta= & \left(C_{A}-C_{S}-C_{L}\right) \sum_{i=0}^{\infty} \pi_{i, 0}-C_{L} \theta \sum_{i=0}^{\infty} \pi_{i, 0} \\
= & \left(C_{A}-C_{S}-C_{L}(1+\theta)\right) \\
& \times\left(1-\left(\frac{\lambda_{\mathrm{pu}}}{\lambda_{\mathrm{pu}}+\mu_{\mathrm{pu}} \bar{\lambda}_{\mathrm{pu}}}+\frac{\lambda_{\mathrm{su}}}{\mu_{\mathrm{su}}}\right)\right),
\end{aligned}
$$

where $\sum_{i=0}^{\infty} \pi_{i, 0}$ means the probability that the BS is not occupied, $C_{A}$ and $C_{S}$ are the energy consumptions per slot when the BS is in the awake state and the sleep state, respectively, and $C_{L}$ is the energy consumption for each listening procedure.

\section{Numerical Results and Discussions}

In this section, we first describe our experiment environment and then discuss the numerical results. To investigate the system performance of CRNs with sleep mode for different sleeping parameters as well as the arrival rates of PU and SU packets, we take the parameters specified of the IEEE $802.11 \mathrm{n}$ in [21].

We assume that every IEEE 802.11n user independently generates its data packet. Referencing to [21], we set the parameters in Table 1.

Figure 1 shows how the average latency $\bar{W}_{\text {su }}$ of SU packets changes versus the sleeping parameter $\theta$ for given arrival rates $\lambda_{\mathrm{su}}$ of SU packets and $\lambda_{\mathrm{pu}}$ of PU packets.

By observing Figure 1, we find that, for the same arrival rates $\lambda_{\text {pu }}$ of PU packets and $\lambda_{\text {su }}$ of SU packets, the average latency $\bar{W}_{\text {su }}$ of SU packets will decrease as the sleeping 


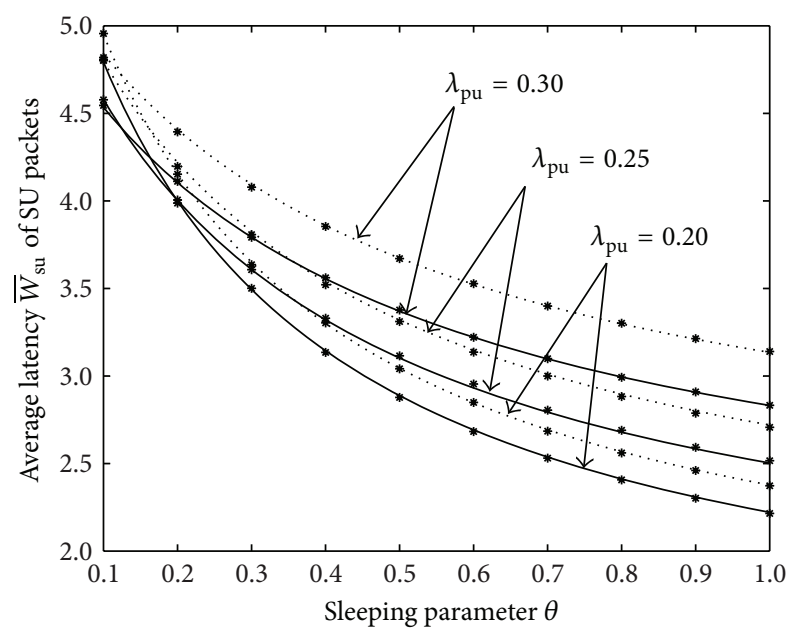

$$
\begin{array}{cc}
\ldots & \lambda_{\mathrm{su}}=0.10 \\
\ldots \ldots & \lambda_{\mathrm{su}}=0.15 \\
* & \text { Simulation }
\end{array}
$$

FIGURE 1: Average latency $\bar{W}_{\text {su }}$ of SU packets versus sleeping parameter $\theta$.

parameter $\theta$ increases. The reason is that the larger the sleeping parameter is, the shorter the average sleep timer length is and the earlier a newly arriving SU packet starts to occupy the spectrum, so the average latency of SU packets will decrease.

In addition, when fixing the arrival rate $\lambda_{\text {su }}$ of SU packets, we find that the arrival rate $\lambda_{\text {pu }}$ of PU packets has twofold influence on the average latency $\bar{W}_{\text {su }}$ of SU packets. For a smaller sleeping parameter $\theta$, the average latency $\bar{W}_{\text {su }}$ of SU packets will decrease as the arrival rate $\lambda_{\mathrm{pu}}$ of PU packets increases. The reason is that when the sleeping parameter is smaller, the sleep period is more likely to be terminated due to the arrival of a PU packet; that is, the dominate factor influencing the state transition from sleep state to awake state is the arrival rate of PU packets. The bigger the arrival rate of PU packets is, the smaller the average time length of the sleep period is and the shorter the SU packets will be delayed at the sleep period, so the average latency of SU packets will decrease. However, for a larger sleeping parameter $\theta$, the average latency $\bar{W}_{\text {su }}$ of SU packets will increase as the arrival rate $\lambda_{\mathrm{pu}}$ of PU packets increases. It is because when the sleeping parameter is larger, the sleep period is more likely to be over with the expiration of the sleep timer; that is, the main factor influencing the state transition from sleep state to awake state is the sleeping parameter. The larger the arrival rate of PU packets is, the longer the SU packets will wait at the buffer, so the average latency of SU packets will increase.

From another point of view, we find that for the same arrival rate $\lambda_{\mathrm{pu}}$ of PU packets and the same sleeping parame$\operatorname{ter} \theta$, the larger the arrival rate $\lambda_{\mathrm{su}}$ of SU packets is, the greater the average latency $\bar{W}_{\text {su }}$ of SU packets will be. Obviously, this is because the larger the arrival rate of SU packets is, the more the SU packets join the system for a certain time, so the number of the SU packets waiting in the buffer is greater, and then the average latency of SU packets will increase.

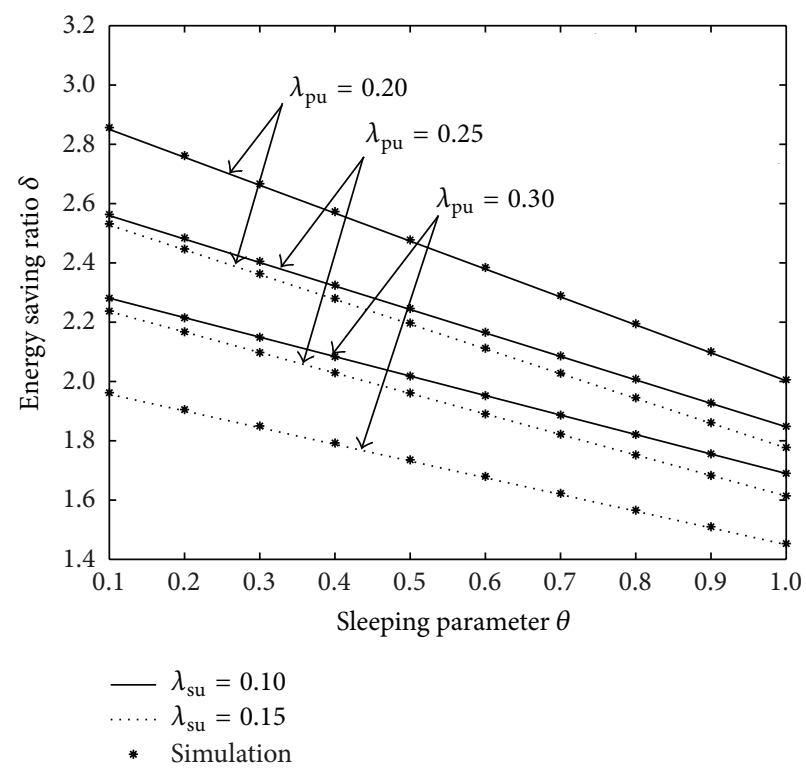

FIGURE 2: Energy saving ratio $\delta$ versus sleeping parameter $\theta$.

Figure 2 illustrates how the energy saving ratio $\delta$ changes versus the sleeping parameter $\theta$ for different arrival rates $\lambda_{\text {su }}$ of SU packets and $\lambda_{\text {pu }}$ of PU packets.

From Figure 2, we find that, for the same arrival rates $\lambda_{\mathrm{pu}}$ of PU packets and $\lambda_{\mathrm{su}}$ of SU packets, the energy saving ratio $\delta$ will decrease as the sleeping parameter $\theta$ increases. It is because the larger the sleeping parameter is, the shorter the average time length of a sleep period is and the less the energy is saved. On the other hand, we also find that for the same sleeping parameter $\theta$, the energy saving ratio $\delta$ will increase as the arrival rates $\lambda_{\mathrm{pu}}$ of PU packets and $\lambda_{\mathrm{su}}$ of SU packets decrease. The reason is that the smaller the arrival rates of PU and SU packets are, the more possible the fact that the sleep period is over with the expiration of the sleep timer is and so the longer the PU and SU packets will be delayed at the sleep period, and then the energy saving ratio will increase.

Comparing the results shown in Figures 1 and 2, we get the conclusion that a greater sleeping parameter will result in not only a smaller average latency of SU packets but also a smaller energy saving ratio; a smaller sleeping parameter will result in not only a greater average latency of SU packets but also a larger energy saving ratio. That is to say, when designing the sleeping timer length in the proposed energy saving strategy, we should balance the average latency of SUs and the energy saving ratio of the system.

\section{Performance Optimization and Pricing Policy}

All the SU packets have their own interests and independently make their decisions to access the spectrum; in other words, they are selfish. Each packet has a goal to maximize its net benefit. In this section, we firstly investigate the individually optimal behavior and the socially optimal behavior of SU packets, respectively. Then, in order to maximize the social 
profit, we impose an appropriate admission fee to SU packets with a pricing policy.

In order to analyze the individually optimal behavior and socially optimal behavior of SU packets in the proposed strategy, some assumptions are given as follows:

(1) Before an SU packet decides to join the buffer, it does not have any information about the system, such as the number of SU packets already in the buffer and the spectrum state.

(2) The reward for an SU packet to be transmitted successfully is $G$.

(3) The cost for an SU packet staying in the system is $C$ per unit of time.

(4) Net-benefit functions of individual SU packets are identical and additive.

(5) The service discipline of SU packets is First Come First Served (FCFS).

\subsection{Performance Optimization}

6.1.1. Individually Optimal Behavior. Once an SU packet joins the buffer, the SU packet will be not able to leave the system until it is transmitted successfully. The SUs are risk neutral; that is to say, they will maximize the expected net benefit. The individually optimal behavior based on the system model built in Section 3.2 is straight forward. The netbenefit function $U_{i}\left(\lambda_{\text {su }}\right)$ of an SU packet is given as follows:

$$
U_{i}\left(\lambda_{\mathrm{su}}\right)=G-C \bar{W}_{\mathrm{su}} .
$$

In order to guarantee the system to be in steady state, we set the lowest arrival rate as $\lambda_{\min }\left(\lambda_{\min } \geq 0\right)$ and the highest arrival rate as $\lambda_{\max }$. Under this constraint, as the arrival rate $\lambda_{\text {su }}$ of SU packets increases, the average latency $\bar{W}_{\text {su }}$ of SU packets grows and the net benefit of an SU packet decreases. Because all the SUs are risk neutral, they all try their best to access the spectrum. Under the condition of $U_{i}\left(\lambda_{\text {su }}\right) \geq 0$, the arrival rate of SU packets will be as large as possible. With the individually optimal arrival rate $\lambda_{\text {su }}^{e}$, SUs will reach an equilibrium state. For this case, no SU packet has any incentive to deviate unilaterally from the current equilibrium state.

We discuss the individually optimal behavior of SU packets in the proposed strategy within the closed interval $\left[\lambda_{\min }, \lambda_{\max }\right]$ as follows:

(1) If $U_{i}\left(\lambda_{\min }\right) \leq 0$, the net-benefit function $U_{i}\left(\lambda_{\mathrm{su}}\right)$ of an $S U$ packet is negative. For this case, the net-benefit function $U_{i}\left(\lambda_{\mathrm{su}}\right)$ of an SU packet is always less than zero. Therefore, $\lambda_{\mathrm{su}}^{e}=\lambda_{\min }$ is an individually optimal arrival rate of SU packets.

(2) If $U_{i}\left(\lambda_{\max }\right) \geq 0$, the net-benefit function $U_{i}\left(\lambda_{\text {su }}\right)$ of an $S U$ packet is positive. For this case, the net-benefit function $U_{i}\left(\lambda_{\mathrm{su}}\right)$ of an SU packet is always more than zero. Therefore, $\lambda_{\mathrm{su}}^{e}=\lambda_{\max }$ is an individually optimal arrival rate of $S U$ packets.

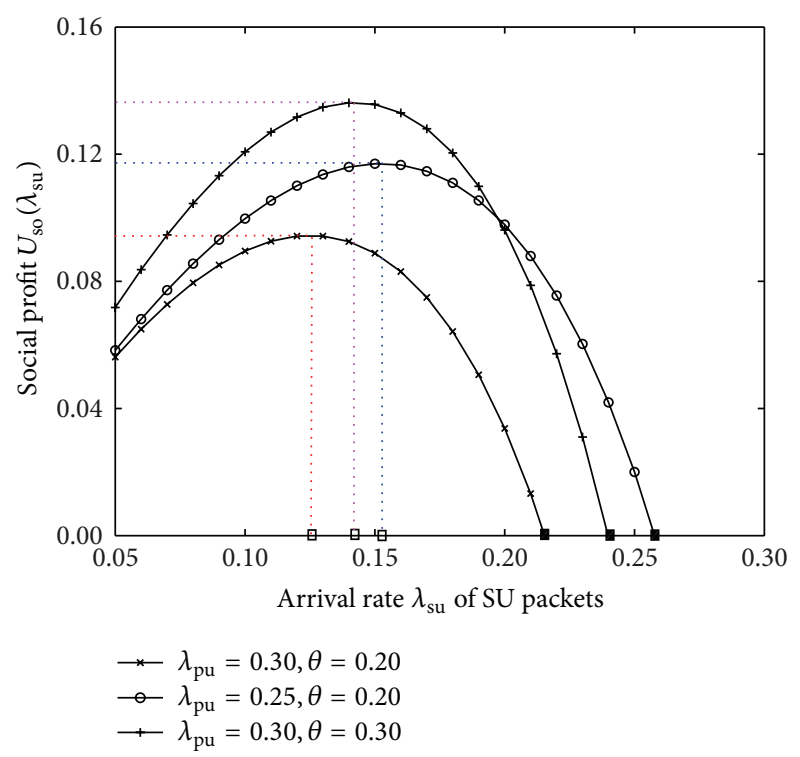

FIGURE 3: Social profit $U_{\text {so }}\left(\lambda_{\text {su }}\right)$ versus arrival rate $\lambda_{\text {su }}$ of SU packets.

(3) For the case of $U_{i}\left(\lambda_{\min }\right)>U_{i}\left(\lambda_{\text {su }}\right)>U_{i}\left(\lambda_{\max }\right)$, if $\lambda_{\mathrm{su}}^{e}=\lambda_{\mathrm{max}}$, then an SU packet which joins the buffer will suffer a negative benefit. Hence, this cannot be an individually optimal arrival rate. Likewise, if $\lambda_{\mathrm{su}}^{e}=\lambda_{\min }$, an SU packet which joins the buffer will get a positive benefit, more than by balking. Hence, this cannot be an individually optimal arrival rate too. Therefore, there exists a unique individually optimal arrival rate where $\lambda_{\mathrm{su}}^{e}$ is subject to $U_{i}\left(\lambda_{\mathrm{su}}^{e}\right)=0$.

6.1.2. Socially Optimal Behavior. Now, we turn our attention to social optimization. The system performance should also be considered from a social point of view. In the case that SU packets are free to occupy the spectrum, by aggregating the net benefits of all the SU packets in the system, the social profit $U_{\text {so }}\left(\lambda_{\text {su }}\right)$ can be given as follows:

$$
U_{\text {so }}\left(\lambda_{\text {su }}\right)=\lambda_{\text {su }}\left(U_{i}\left(\lambda_{\text {su }}\right)\right)=\lambda_{\text {su }}\left(G-C \bar{W}_{\text {su }}\right) .
$$

By maximizing the social profit $U_{\mathrm{so}}\left(\lambda_{\mathrm{su}}\right)$ in (21), we can get the socially optimal arrival rate $\lambda_{\mathrm{su}}^{*}$ as follows:

$$
\lambda_{\mathrm{su}}^{*}=\underset{\lambda_{\min } \leq \lambda_{\mathrm{su}} \leq \lambda_{\max }}{\arg \max }\left\{\lambda_{\mathrm{su}}\left(G-C \bar{W}_{\mathrm{su}}\right)\right\},
$$

where "arg max" stands for the argument of the maximum, in other words, the set of points from which the given function $U_{\text {so }}\left(\lambda_{\text {su }}\right)$ gets maximum.

Employing the parameters used in Table 1 and setting $G=5$ and $C=1$ as an example, we show how the social profit $U_{\text {so }}\left(\lambda_{\text {su }}\right)$ changes with respect to the arrival rate $\lambda_{\text {su }}$ of SU packets in Figure 3.

In Figure 3, we observe that when the arrival rate $\lambda_{\text {su }}$ of SU packets is smaller, with the increase in the arrival rate $\lambda_{\text {su }}$ of SU packets, the social profit $U_{\text {so }}\left(\lambda_{\text {su }}\right)$ shows a rising trend; when the arrival rate $\lambda_{\text {su }}$ of SU packets is greater, with the increase in the arrival rate $\lambda_{\text {su }}$ of SU packets, the social profit 
$U_{\text {so }}\left(\lambda_{\text {su }}\right)$ shows a downward trend. Therefore, there exist a socially optimal arrival rate $\lambda_{\mathrm{su}}^{*}$ and a maximum social profit.

We note that the social profit $U_{\text {so }}\left(\lambda_{\text {su }}\right)=0$ means that the net benefit of an SU packet is also equal to zero; that is, $U_{i}\left(\lambda_{\text {su }}\right)=0$. For this case, by setting the social profit $U_{\text {so }}\left(\lambda_{\text {su }}\right)=0$, we can also obtain the individually optimal arrival rate $\lambda_{\text {su. }}^{e}$.

In Figure 3, the socially optimal arrival rates $\lambda_{\mathrm{su}}^{*}$ are marked with " $\square$ " and the individually optimal arrival rates $\lambda_{\mathrm{su}}^{e}$ are marked with " $\square$ ". Obviously, $\lambda_{\mathrm{su}}^{*} \leq \lambda_{\mathrm{su}}^{e}$. This means that there are more SU packets to join the buffer under individually optimal behavior. In order to restrain the individually optimal arrival rate $\lambda_{\mathrm{su}}^{e}$ and make it equal to the socially optimal arrival rate $\lambda_{\mathrm{su}}^{*}$, we impose an appropriate admission fee to SU packets.

6.2. Pricing Policy. In order to present a reasonable pricing policy, we should firstly search the socially optimal arrival rate $\lambda_{\mathrm{su}}^{*}$ of SU packets.

6.2.1. Optimization Algorithm. Since the social profit $U_{\text {so }}\left(\lambda_{\mathrm{su}}\right)$ in (21) is nonlinear and continuous, it is difficult to give the optimally arrival rate $\lambda_{\mathrm{su}}^{*}$ of SU packets mathematically. We turn to an intelligent optimization method named SPSO [22]. In the SPSO process, the velocity of each particle is iteratively adjusted so that the particle stochastically oscillates around the local optimum and global optimum locations. Based on the idea of SPSO, we put forward an algorithm to obtain the socially optimal arrival rate $\lambda_{\text {su }}^{*}$ of SU packets in Algorithm 2.

Algorithm 2 (algorithm to obtain the socially optimal arrival rate $\left.\lambda_{\mathrm{su}}^{*}\right)$.

Step 1. Initialize SU packets number $N$, upper bound $\lambda_{\mathrm{su}}^{\text {up }}$ for the arrival rate of SU packets, lower bound $\lambda_{\mathrm{su}}^{\text {low }}$ for the arrival rate of SU packets, acceleration constants $c_{1}$ and $c_{2}$ within $(0,2)$, minimum value $\omega_{\min }$ and maximum value $\omega_{\max }$ of the inertia weight, maximum number iter $_{\max }$ of iterations, and maximum value $V_{\mathrm{su}}^{\mathrm{max}}$ of the velocity.

Step 2. Randomly set initial arrival rate $\left(\lambda_{\mathrm{su}}^{i}\right) \in\left[\lambda_{\mathrm{su}}^{\text {low }}, \lambda_{\mathrm{su}}^{\mathrm{up}}\right]$ and initial velocity $\left(v_{\mathrm{su}}^{i}\right) \in[0,1]$ for $i$ th $(i=1,2, \ldots, N) \mathrm{SU}$ packet. Set the initial number of iterations by iter $=0$.

Step 3. For each SU packet with arrival rate $\lambda_{\mathrm{su}}^{i}(i=1,2, \ldots$, $N)$, calculate the social profit by

$$
U_{\mathrm{so}}^{i}=\lambda_{\mathrm{su}}^{i}\left(G-C \bar{W}_{\mathrm{su}}^{i}\right)
$$

where $\bar{W}_{\text {su }}^{i}$ is the average latency of SU packets with the arrival rate $\lambda_{\mathrm{su}}^{i}$.

Step 4. Search the previous maximum social profit $\max _{\text {so }}^{i}$ and the previous socially optimal arrival rate $p_{\mathrm{su}}^{i}$ for the $i$ th $(i=$ $1,2, \ldots, N)$ SU packet.
Step 5. Identify the SU packet in the search space with maximum social profit and assign its arrival rate to the variable $p_{\text {su }}^{g}$ by

$$
p_{\mathrm{su}}^{g}=\underset{i=1,2, \ldots, N}{\arg \max }\left\{\max _{\mathrm{so}}^{i}\right\}
$$

Step 6. Calculate the inertia weight $\omega$ by

$$
\omega=\omega_{\max }-\frac{\omega_{\max }-\omega_{\min }}{\text { iter }_{\max }} * \text { iter. }
$$

Step 7. Update the velocity and the arrival rate of the $i$ th $(i=$ $1,2, \ldots, N)$ SU packet by

$$
\begin{aligned}
& v_{\mathrm{su}}^{i}=\omega v_{\mathrm{su}}^{i}+c_{1} r_{1}\left(p_{\mathrm{su}}^{i}-\lambda_{\mathrm{su}}^{i}\right)+c_{2} r_{2}\left(p_{\mathrm{su}}^{g}-\lambda_{\mathrm{su}}^{i}\right), \\
& \lambda_{\mathrm{su}}^{i}=\lambda_{\mathrm{su}}^{i}+v_{\mathrm{su}}^{i},
\end{aligned}
$$

where $r_{1}, r_{2} \in U(0,1) . r_{1}$ and $r_{2}$ are uniform random variables. $v_{\mathrm{su}}^{i}$ is kept within the range $\left[-V_{\mathrm{su}}^{\max },+V_{\mathrm{su}}^{\max }\right]$.

Step 8. Update the number of iterations by iter $=$ iter +1 .

$$
\begin{aligned}
& \text { If }\left\{\text { iter } \leq \text { iter }_{\max }\right\} \text {, then goto Step } 3 . \\
& \text { Else } \lambda_{\mathrm{su}}^{*}=p_{\mathrm{su}}^{g} .
\end{aligned}
$$

Step 9. Output $\lambda_{\mathrm{su}}^{*}$.

6.2.2. Admission Fee. When an admission fee $p$ is imposed, the modified net-benefit function $U_{i}^{\prime}\left(\lambda_{\mathrm{su}}\right)$ of an SU packet is given as follows:

$$
U_{i}^{\prime}\left(\lambda_{\mathrm{su}}\right)=G-p-C \bar{W}_{\mathrm{su}}
$$

while the modified social profit $U_{\text {so }}^{\prime}\left(\lambda_{\text {su }}\right)$ is given as follows:

$$
\begin{aligned}
U_{\text {so }}^{\prime}\left(\lambda_{\text {su }}\right) & =\lambda_{\text {su }}\left(G-p-C \bar{W}_{\text {su }}\right)+\lambda_{\text {su }} p \\
& =\lambda_{\text {su }}\left(G-C \bar{W}_{\text {su }}\right) .
\end{aligned}
$$

Comparing (21) and (28), we find that the admission fee has no effect on the social profit. That is to say, with the admission fee $p$, the socially optimal arrival rate $\lambda_{\mathrm{su}}^{*}$ and the maximum social profit will not be changed.

Substituting $\lambda_{\text {su }}^{*}$ obtained in the Algorithm 2 into (27) and letting $U_{i}^{\prime}\left(\lambda_{\text {su }}^{*}\right)=0$, we can obtain the admission fee $p$ to SU packets as follows:

$$
p=G-C \bar{W}_{\mathrm{su}}^{*}
$$

where $\bar{W}_{\text {su }}^{*}$ is the average latency of SU packets with the socially optimal arrival rate $\lambda_{\mathrm{su}}^{*}$.

For different arrival rates $\lambda_{\mathrm{pu}}$ of PU packets and sleeping parameters $\theta$, we summarize the numerical results for socially optimal arrival rate $\lambda_{\text {su }}^{*}$ of SU packets and the admission fee $p$ to SU packets in Table 2 . 
TABLE 2: Numerical results of the pricing policy.

\begin{tabular}{lccc}
\hline $\begin{array}{l}\text { Arrival rate } \\
\lambda_{\text {pu }} \text { of PU } \\
\text { packets }\end{array}$ & $\begin{array}{c}\text { Sleeping } \\
\text { parameter } \theta\end{array}$ & $\begin{array}{c}\text { Socially } \\
\text { optimal } \\
\text { arrival rate } \\
\lambda_{\text {su }}^{*} \text { of SU } \\
\text { packets }\end{array}$ & $\begin{array}{c}\text { Admission } \\
\text { fee } p \text { to SU } \\
\text { packets }\end{array}$ \\
\hline \multirow{2}{*}{0.20} & 0.30 & 0.2144 & 1.0792 \\
& 0.40 & 0.2335 & 1.3267 \\
0.25 & 0.50 & 0.2453 & 1.5135 \\
\hline \multirow{3}{*}{0.30} & 0.30 & 0.1766 & 1.0313 \\
& 0.40 & 0.1913 & 1.2284 \\
& 0.50 & 0.2012 & 1.3810 \\
\hline
\end{tabular}

From Table 2, we observe that when the arrival rate $\lambda_{\text {pu }}$ of $\mathrm{PU}$ packets is given, as the sleeping parameter $\theta$ increases, the admission fee $p$ to SU packets will increase. This is because the greater the sleeping parameter is, the shorter the average time length of a sleep period is, the shorter the average latency of SU packets is, and the more SU packets will try to join the system. So, in order to control the number of SU packets waiting in the buffer and guarantee the maximum social profit, the admission fee to SU packets should be set higher. On the other hand, we find that when the sleeping parameter $\theta$ is given, as the arrival rate $\lambda_{\mathrm{pu}}$ of PU packets increases, the admission fee $p$ to SU packets will decrease. The reason is that the greater the arrival rate of PU packets is, the longer the SU packets will wait at the buffer, the larger the average latency of SU packets is, and the less the SU packets will try to join the system. For this case, in order to encourage more SU packets to join the system and obtain the maximum social profit, the admission fee to SU packets should be set lower.

\section{Conclusions}

In this paper, we firstly introduced an energy saving strategy in CRNs. By building a QBD process model and using the Matrix-Geometric Solution method, we presented the stability condition of the system and analyzed the performance measures, such as the average latency of SU packets and the energy saving ratio of the system in steady state. With the help of the natural reward-cost structure, we studied the individually optimal behavior and the socially optimal behavior of SU packets in the proposed energy saving strategy. Moreover, we searched the unique socially optimal arrival rate of SU packets by using an optimization algorithm based on SPSO method. By comparing the individually optimal behavior and the socially optimal behavior, we imposed an appropriate admission fee to SU packets. With the numerical results, we illustrated the impacts of system parameters on the system performance and the pricing policy.

In this paper, we considered a CRN with one BS and homogeneous SU packets. In future work, we will construct a new system model to capture the energy saving strategy concerning multihop CRNs and heterogenous SU packets.

\section{Competing Interests}

The authors declare that there are no competing interests regarding the publication of this paper.

\section{Acknowledgments}

This work was supported in part by National Natural Science Foundation (no. 61472342), China.

\section{References}

[1] K. Shin, H. Kim, A. Min, and A. Kumar, "Cognitive radios for dynamic spectrum access: from concept to reality," IEEE Wireless Communications, vol. 17, no. 6, pp. 64-74, 2010.

[2] X. Huang, T. Han, and N. Ansari, "On green-energy-powered cognitive radio networks," IEEE Communications Surveys and Tutorials, vol. 17, no. 2, pp. 827-842, 2015.

[3] P. Dini, M. Miozzo, N. Bui, and N. Baldo, "A model to analyze the energy savings of base station sleep mode in LTE HetNets," in Proceedings of the IEEE International Conference on Green Computing and Communications and IEEE Internet of Things and IEEE Cyber, Physical and Social Computing (GreenComiThings-CPSCom '13), pp. 1375-1380, IEEE, Beijing, China, August 2013.

[4] B. Rengarajan, G. Rizzo, and M. A. Marsan, "Energy-optimal base station density in cellular access networks with sleep modes," Computer Networks, vol. 78, pp. 152-163, 2015.

[5] Y.-C. Liang, K.-C. Chen, G. Y. Li, and P. Mähönen, "Cognitive radio networking and communications: an overview," IEEE Transactions on Vehicular Technology, vol. 60, no. 7, pp. 33863407, 2011.

[6] Y. Zhao, S. Jin, and W. Yue, "An adjustable channel bonding strategy in centralized cognitive radio networks and its performance optimization," Quality Technology and Quantitative Management, vol. 12, no. 3, pp. 291-310, 2015.

[7] H. Li and Z. Han, "Socially optimal queuing control in cognitive radio networks subject to service interruptions: to queue or not to queue?" IEEE Transactions on Wireless Communications, vol. 10, no. 5, pp. 1656-1666, 2011.

[8] J. Li, P. Vetter, C. A. Chan et al., "Sleep mode mechanism with improved upstream performance for passive optical networks," in Proceedings of the IEEE International Conference on Communications (ICC '14), pp. 3871-3876, Sydney, Australia, June 2014.

[9] S. Baek, J. J. Son, and B. D. Choi, "Performance analysis of push-to-talk over IEEE 802.16e with sleep mode and idle mode," Telecommunication Systems, vol. 47, no. 3-4, pp. 291-302, 2011.

[10] S. De Vuyst, K. De Turck, D. Fiems, S. Wittevrongel, and $\mathrm{H}$. Bruneel, "Delay versus energy consumption of the IEEE 802.16e sleep-mode mechanism," IEEE Transactions on Wireless Communications, vol. 8, no. 11, pp. 5383-5387, 2009.

[11] J. Premalatha, U. Anitha, V. Manonmani, and P. Ganesan, "Survey on energy saving methods for green communication network," Indian Journal of Science \& Technology, vol. 8, no. 19, pp. 1-5, 2015.

[12] G. Ghosh, P. Das, and S. Chatterjee, "Cognitive radio and dynamic spectrum access-a study," International Journal of Next-Generation Networks, vol. 6, no. 1, pp. 43-60, 2014. 
[13] S.-C. Lin and K.-C. Chen, "Spectrum aware opportunistic routing in cognitive radio networks," in Proceedings of the 53rd IEEE Global Communications Conference (GLOBECOM '10), pp. 1-6, December 2010.

[14] A. H. Anwar, A. El Shafie, A. Mohamed, T. Elbatt, and M. Guizani, "Interference-based optimal power-efficient access scheme for cognitive radio networks," in Proceedings of the International Conference on Computing, Networking and Communications (ICNC '15), pp. 962-967, Garden Grove, Calif, USA, February 2015.

[15] A. Ö. Ercan, M. O. Sunay, and S. Pollin, "Analysis of energy harvesting for green cognitive radio networks," in Proceedings of the 1st International Workshop on Cognitive Cellular Systems (CCS '14), pp. 1-7, IEEE, September 2014.

[16] R. N. Yadav, R. Misra, U. Gupta, and S. Bhagat, "Opportunistic spectrum access in CR network in licensed and unlicensed channels," in Proceedings of the International Conference on Distributed Computing and Networking (ICDCN '15), pp. 39-47, ACM, Goa, India, January 2015.

[17] C. T. Do, N. H. Tran, M. Van Nguyen, C. S. Hong, and S. Lee, "Social optimization strategy in unobserved queueing systems in cognitive radio networks," IEEE Communications Letters, vol. 16, no. 12, pp. 1944-1947, 2012.

[18] J. Zheng, Y. Cai, X. Chen, R. Li, and H. Zhang, “Optimal base station sleeping in green cellular networks: a distributed cooperative framework based on game theory," IEEE Transactions on Wireless Communications, vol. 14, no. 8, pp. 4391-4406, 2015.

[19] N. H. Tran, C. S. Hong, Z. Han, and S. Lee, "Optimal pricing effect on equilibrium behaviors of delay-sensitive users in cognitive radio networks," IEEE Journal on Selected Areas in Communications, vol. 31, no. 11, pp. 2566-2579, 2013.

[20] M. F. Neuts, Matrix-Geometric Solutions in Stochastic Models, Johns Hopkins University Press, Baltimore, Md, USA, 1981.

[21] D. Halperin, B. Greenstein, A. Sheth et al., "Demystifying 802.11n power consumption," in Proceedings of the International Conference on Power Aware Computing and Systems (HotPower '10), pp. 1-5, 2010.

[22] R. Poli, J. Kennedy, and T. Blackwell, "Particle swarm optimization," Swarm Intelligence, vol. 1, no. 1, pp. 33-57, 2007. 

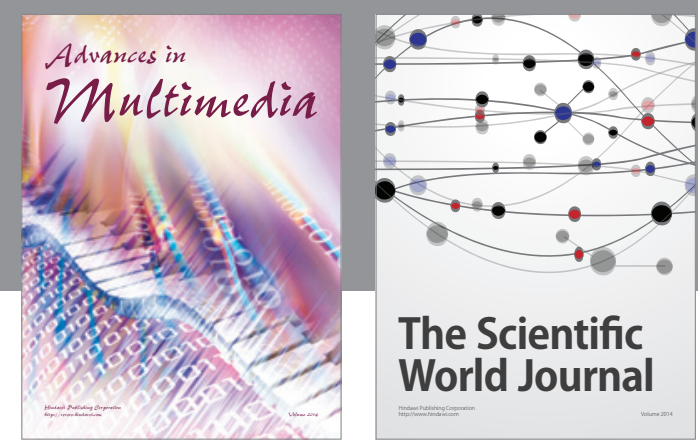

The Scientific World Journal
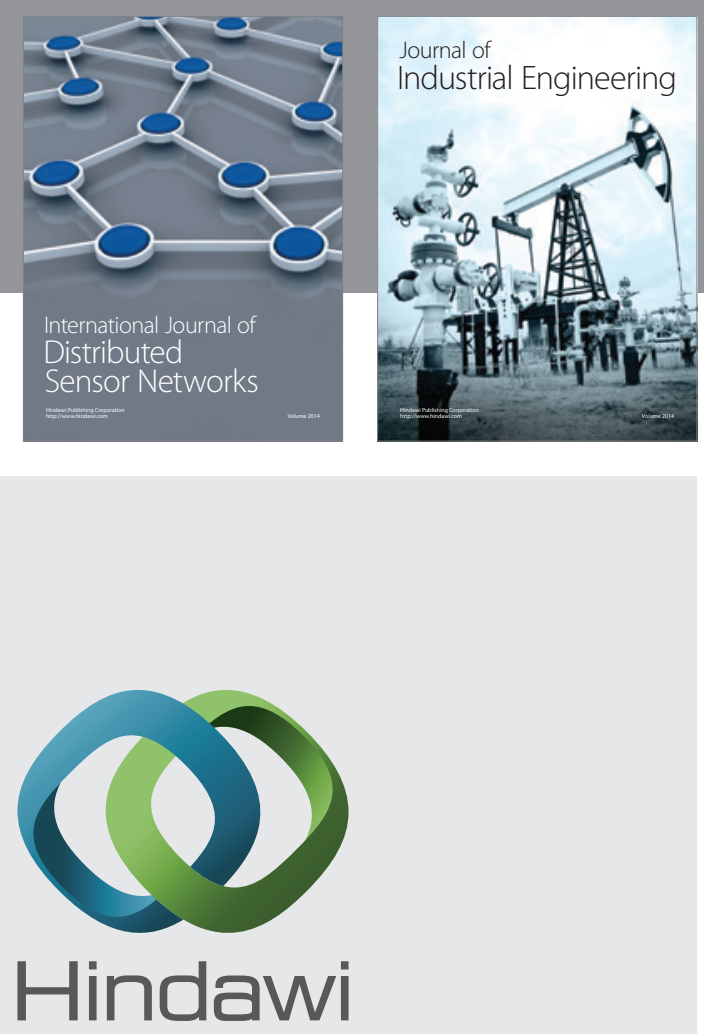

Submit your manuscripts at

http://www.hindawi.com

\section{Computer Networks} and Communications
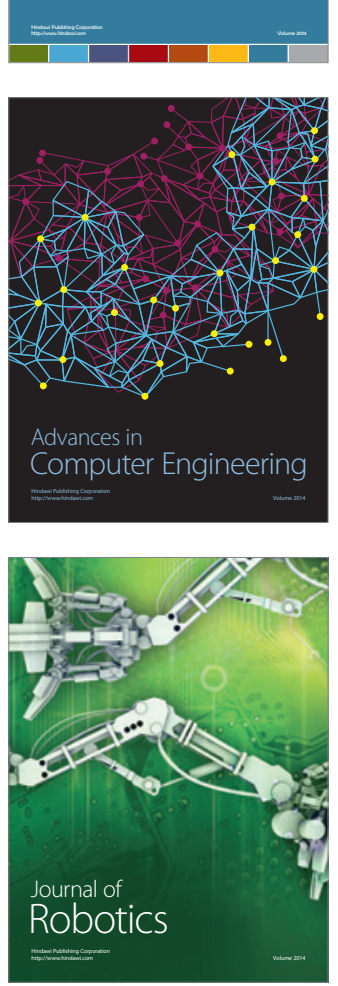
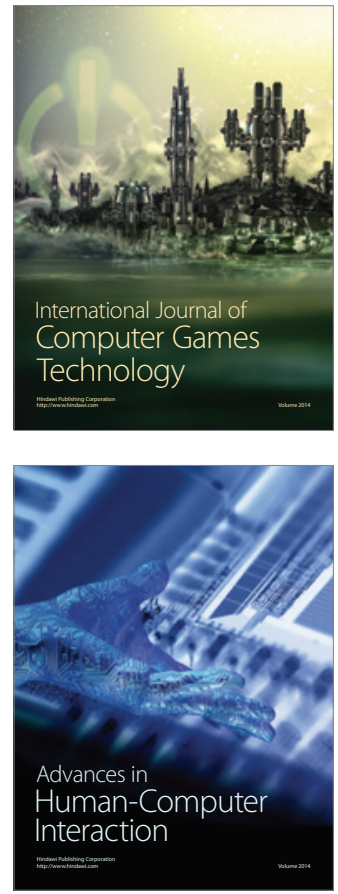
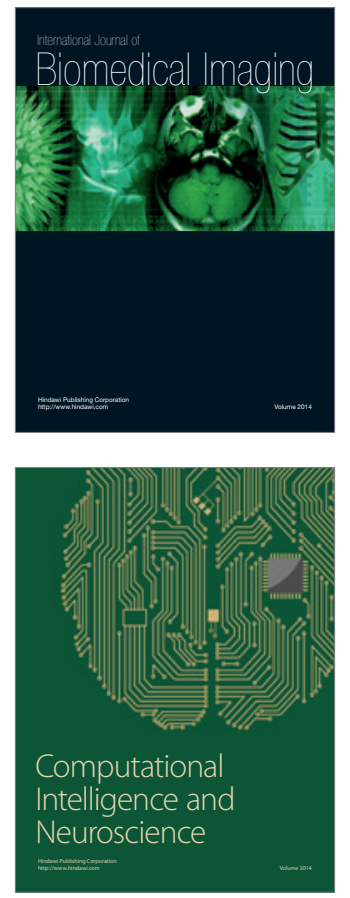
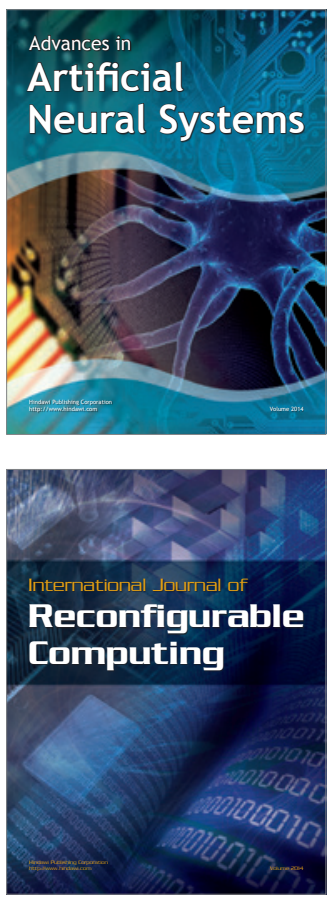
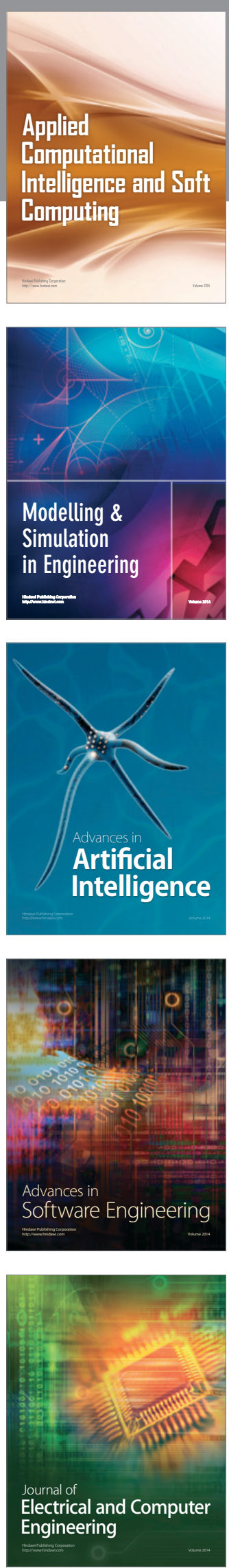